Nonlin. Processes Geophys., 25, 77-88, 2018

https://doi.org/10.5194/npg-25-77-2018

(C) Author(s) 2018. This work is distributed under

the Creative Commons Attribution 4.0 License.

\title{
Derivation of the entropic formula for the statistical mechanics of space plasmas
}

\author{
George Livadiotis \\ Southwest Research Institute, Space Science and Engineering, San Antonio, TX, USA \\ Correspondence: George Livadiotis (glivadiotis@ swri.edu)
}

Received: 19 September 2017 - Discussion started: 25 September 2017

Revised: 16 December 2017 - Accepted: 1 January 2018 - Published: 7 February 2018

\begin{abstract}
Kappa distributions describe velocities and energies of plasma populations in space plasmas. The statistical origin of these distributions is associated with the framework of nonextensive statistical mechanics. Indeed, the kappa distribution is derived by maximizing the $q$ entropy of Tsallis, under the constraints of the canonical ensemble. However, the question remains as to what the physical origin of this entropic formulation is. This paper shows that the $q$ entropy can be derived by adapting the additivity of energy and entropy.
\end{abstract}

\section{Introduction}

Space plasmas are collisionless and correlated particle systems characterized by a non-Maxwellian behavior, typically described by the formulations of kappa distributions. The origin of this vastly different statistical behavior between classical systems and space plasmas is the manifestation of correlations between the plasma particles. These systems are characterized by long-range interactions that induce correlations, resulting in a collective behavior among particles (e.g., see Jund et al., 1995; Salazar and Toral, 1999; Villain, 2008; Tsallis, 2009; Grassi, 2010; Tirnakli and Borges, 2016). The induction of any type of correlations among particles (more accurately, among particle energies or particle phase space) departs the system from thermal equilibrium to be restabilized into other stationary states out of thermal equilibrium described by kappa distributions, or combinations/superposition thereof. (Note that as we will see in Sect. 3, single kappa distributions induce a certain type of correlation, which, however, can be further generalized when a combination or superposition of kappa distributions is taken into account; e.g., see Spectral Statistics, Tsallis, 2009, Linear/Nonlinear superposition, chap. 6.2.1; Livadiotis and McComas, 2013a, Appendix A; Livadiotis, 2017a, chap. 4.3.4.)

Kappa distributions describe numerous space plasma populations. Several examples are the following: (i) the inner heliosphere, including solar wind (e.g., Maksimovic et al., 1997; Pierrard et al., 1999; Mann et al., 2002; Marsch, 2006; Zouganelis, 2008; Štverák et al., 2009; Livadiotis and McComas, 2013a; Yoon, 2014; Pierrard and Pieters, 2015; Pavlos et al., 2016), solar spectra (e.g., Dzifčáková and Dudík, 2013; Dzifčáková, et al., 2015), solar corona (e.g., Owocki and Scudder, 1983; Vocks et al., 2008; Lee et al., 2013; Cranmer, 2014), solar energetic particles (e.g., Xiao et al., 2008; Laming et al., 2013), corotating interaction regions (e.g., Chotoo et al., 2000), and related solar flares (e.g., Mann et al., 2009; Livadiotis and McComas, 2013b; Bian et al., 2014; Jeffrey et al., 2016); (ii) planetary magnetospheres, including magnetosheath (e.g., Formisano et al., 1973; Ogasawara et al., 2013), magnetopause (e.g., Ogasawara et al., 2015), magnetotail (e.g., Grabbe, 2000), ring current (e.g., Pisarenko et al., 2002), plasma sheet (e.g., Christon, 1987; Wang et al., 2003; Kletzing et al., 2003), magnetospheric substorms (e.g., Hapgood et al., 2011), aurora (e.g., Ogasawara et al., 2017), magnetospheres of giant planets, such as Jovian (e.g., Collier and Hamilton, 1995; Mauk et al., 2004), Saturnian (e.g., Dialynas et al., 2009; Livi et al., 2014; Carbary et al., 2014), Uranian (e.g., Mauk et al., 1987), and Neptunian (Krimigis et al., 1989), magnetospheres of planetary moons, such as Io (e.g., Moncuquet et al., 2002) and Enceladus (e.g., Jurac et al., 2002), and cometary magnetospheres (e.g., Broiles et al.,, 2016a, b); (iii) the outer heliosphere and the inner heliosheath (e.g., Decker and Krimigis, 2003; 
Decker et al., 2005; Heerikhuisen et al., 2008, 2015; Zank et al., 2010; Livadiotis et al., 2011, 2012; 2013; Livadiotis and McComas, 2011a, 2012; 2013c; Livadiotis, 2014, 2016; Fuselier et al., 2014; Zirnstein and McComas, 2015; Zank, 2015); (iv) beyond the heliosphere, including HII regions (e.g., Nicholls et al., 2012), planetary nebula (e.g., Nicholls et al., 2013; Zhang et al., 2014), and supernova magnetospheres (e.g., Raymond et al., 2010), and on cosmological scales (e.g., Hou et al., 2017); and (v) other space-plasmarelated analyses (e.g., Milovanov and Zelenyi, 2000; Saito et al., 2000; Du, 2004; Yoon et al., 2006, 2012; Raadu and Shafiq, 2007; Livadiotis, 2009, 2015a, b, c, 2018; Tribeche et al., 2009; Hellberg et al., 2009; Livadiotis and McComas, 2010b, 2014; Baluku et al., 2010; Le Roux et al., 2010; Eslami et al., 2011; Kourakis et al., 2012; Randol and Christian, 2014, 2016; Varotsos et al., 2014; Fisk and Gloeckler, 2014; Viñas et al., 2014, 2015; Ourabah et al., 2015; Dos Santos et al., 2016; Nicolaou and Livadiotis, 2016). (See also the book Livadiotis, 2017a, and references therein.) Finally, it has to be noted that the kappa distributions and its associated statistical mechanics have been applied in a variety of disciplines other than space and plasma physics. A few examples are the following: in sociometry, e.g., the internet (Abe and Suzuki, 2003) and in citation networks of scientific papers (Tsallis and De Albuquerque, 2000) and urban agglomeration (Malacarne et al., 2001); in linguistics (Montemurro, 2001); in economics (Borland, 2002); in biochemistry (Andricioaei and Straub, 1996); in applied statistics (Habeck et al., 2005); in nonlinear dynamics (Borges et al., 2002); in physical chemistry (Livadiotis, 2009); and in ecology (Livadiotis et al., 2015, 2016).

Empirical kappa distributions were introduced in mid1960s by Binsack (1966), Olbert (1968), and Vasyliũnas (1968), while their connection with statistical mechanics was shown and studied in detail about half a century later (see Livadiotis and McComas, 2009, and references therein). In particular, the statistical origin of these distributions is now widely accepted to be determined within the framework of nonextensive statistical mechanics (Tsallis, 2009). This is a consistent generalization of the classical statistical mechanics, which is based on a monoparametric ( $q$ index) entropic formula (Tsallis, 1988). The theoretical $q$ exponential distribution, which results from the maximization of entropy in the canonical ensemble, has the same formulation as the empirical kappa distribution; the two distributions are identical under the transformation of their characteristic indices $(q=1+1 / \kappa)$.

Though a consistent connection of the mathematical model of kappa distributions has been attained with the physical means of entropy maximization, this does not precisely answer the main question regarding the origin of these distributions. We have only shifted the modeling from the distributions to the entropic formulation. Therefore, we may understand now that the statistical origin of kappa distributions is given by the Tsallis entropy maximization in the canonical ensemble, but still, the origin of this specific entropic formulation remains unknown.

Certainly, there are various mechanisms responsible for generating kappa distributions in space and other plasmas, for example, the presence of pickup ions (Livadiotis and McComas, 2010a, 2011a) or weak turbulence (Yoon et al., 2012; Yoon, 2014). Moreover, kappa distributions belong to the framework of nonextensive statistical mechanics. Thus, once a kappa distribution is generated and stabilized into a plasma population, the whole "tool package" of nonextensive statistical mechanics is applicable for describing the statistical physics of this population; for instance, the entropy is given by the Tsallis formulation, while the temperature can be determined by the mean kinetic energy.

Here, we do not argue which mechanisms generate kappa distributions in space plasmas, rather the physical reasons that these distributions sustain themselves in space plasmas once generated. The typical answer is that this is an effect of the presence and preservation of correlations in the collisionless environment that governs space plasmas. (For particle systems such as space plasmas, collisions can destroy correlations, and thus, their collective behavior.) The collisionless environment conserves the energy. Moreover, weakly coupled plasmas (mutual electron and ion potential energy is small compared to the average kinetic energy) can be described as ideal gases. Interparticle energy terms can be ignored, leading to the additivity of energy: the energy of a multi-particle state is the sum of the energies of all the oneparticle states involved. On the other hand, the preservation of local correlations among particles creates a conceptual separation of particles in correlation clusters. Debye spheres are correlation clusters that may include up to trillions of particles since space plasmas are weakly coupled (Bryant, 1996; Rubab and Murtaza, 2006; Gougam and Tribeche, 2011; Livadiotis and McComas, 2014). This structure can lead to the additivity of entropy: the entropy of a multi-particle state is the sum of the entropies of all the one-particle states involved. (Note that ideal gases are considered to have (i) zero interparticle interactions, and (ii) zero particle correlations. While ideal gases are characterized by short-range interactions that cannot induce correlations among particles, space plasmas have interactions weak enough to be negligible but with a long enough range that correlations can be induced.)

The purpose of this paper is to show that there is a deeper connection of Tsallis $q$ entropy and space plasmas: namely, we will show that two simple first principles, such as the additive energy and additive entropy, which apply to plasma particle populations, are sufficient for indicating the specific formula of $q$ entropy (Fig. 1). Therefore, the main objective of this work is to demonstrate the theory which determines that the entropic form given by the $q$-entropy formula proposed originally by Tsallis (1988) follows from certain assumptions regarding the (microscopic) state of the system. The importance of this discussion for the (space) plasma physics community resides mostly in the fact that the kappa 


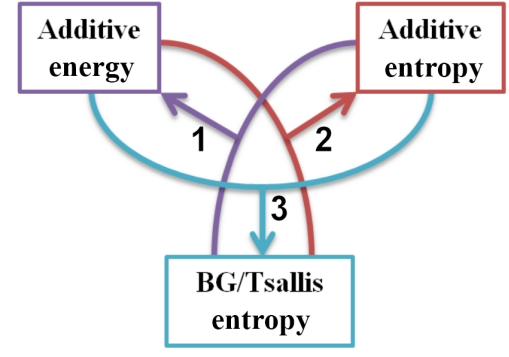

Figure 1. The infographic indicates the following triplet of concepts: (i) additive energy, (ii) additive entropy, and (iii) BG or Tsallis entropic formulation. Given any two out of the three features, the third can be derived. It is already known that BG or Tsallis entropic formulations can lead to additive entropy if the energy is also additive (red arrow 2). In the same way, it can be shown that these entropic formulations can lead to additive energy if the entropy is additive (purple arrow 1). The objective here is to show that the entropic formula can be derived from the additivity of energy and entropy (blue arrow 3).

velocity/energy distribution functions, ubiquitously observed in space and astrophysical environments, can be derived from the maximization of the $q$ entropy, under the constraints of a canonical ensemble.

In Sect. 2, we describe the physical motive of this paper in detail. In Sect. 3, we show in detail a similar property for both the entropic formalisms of Boltzmann-Gibbs (BG) and Tsallis: the entropy is non-additive in general for some arbitrary probability distribution, but it can become additive specifically for the canonical probability distribution (the one that maximizes the corresponding entropy). In Sect. 4, we show how we can determine the entropic formula appropriate for describing the plasma particle populations, simply by setting two first principles' properties, obvious for collisionless plasmas: energy and entropy are additive, at least macroscopically. Finally, Sect. 5 briefly summarizes the conclusions.

\section{Physical motive}

Classical BG statistical mechanics characterizes systems with no correlations among particle velocities or energies. Therefore, the joint two-particle probability distribution can be expressed as the product of the one-particle identical and independent discrete distributions, i.e., labeling the two particles with $\mathrm{A}$ and $\mathrm{B}, p_{i j}^{\mathrm{A}+\mathrm{B}}=p_{i}^{\mathrm{A}} \cdot p_{j}^{\mathrm{B}}$. Hereafter, we consider a particle system described by a discrete energy spectrum $\left\{\varepsilon_{k}\right\}_{k=1}^{W}$, which is associated with a discrete probability distribution $\left\{p_{k}\right\}_{k=1}^{W}$. The same semantics is used when the system is separated in two subsystems, A and B, where the twoparticle distribution describes a two-particle state, with one particle at each subsystem. The logarithm of the probability is an additive function, $\ln p_{i j}^{\mathrm{A}+\mathrm{B}}=\ln p_{i}^{\mathrm{A}}+\ln p_{j}^{\mathrm{B}}$, from which we obtain the additivity of entropy (here the BG entropy), $S^{\mathrm{A}+\mathrm{B}}=S^{\mathrm{A}}+S^{\mathrm{B}}$. For special cases, however, where the in- dependence relationship does not apply, $p_{i j}^{\mathrm{A}+\mathrm{B}} \neq p_{i}^{\mathrm{A}} \cdot p_{j}^{\mathrm{B}}$, the entropy is non-additive, $S^{\mathrm{A}+\mathrm{B}} \neq S^{\mathrm{A}}+S^{\mathrm{B}}$. The logical reciprocant to the statements above is provided by the uniqueness theorem of Shannon (1948) and Khinchin (1957) that showed that under the assumption of the additivity of entropy (and other basic properties of entropy), the sufficient and necessary entropic form is given by the BG formula.

Nonextensive statistical mechanics characterizes systems with correlations among particles, $p_{i j}^{\mathrm{A}+\mathrm{B}} \neq p_{i}^{\mathrm{A}} \cdot p_{j}^{\mathrm{B}}$. For special systems, however, where the independence relationship still applies, the entropy (here the $q$ entropy) is non-additive, $S^{\mathrm{A}+\mathrm{B}} \neq S^{\mathrm{A}}+S^{\mathrm{B}}$; in particular, a square, nonlinear term is added to the summation, $S^{\mathrm{A}+\mathrm{B}}=S^{\mathrm{A}}+S^{\mathrm{B}}+(1-q) S^{\mathrm{A}} S^{\mathrm{B}}$, for some value of the entropic parameter $q$ (where we set the Boltzmann constant $k_{\mathrm{B}}$ to 1 ). Note that the logical reciprocant also exists for this case, as shown by Dos Santos (1997) and Abe (2000); namely, under the assumption of the nonadditive property mentioned (and other basic properties of entropy), the sufficient and necessary entropic form is given by the $q$ entropic formula of Tsallis (1988).

Another property that is related with the additivity but is even more subtle and difficult to ascertain is the extensivity of the entropy. A non-additive entropy may be assumed to be also nonextensive, but it is the inverse assumption that is always correct; i.e., nonextensivity implies non-additivity. Nevertheless, certain correlations, expressed by the probability relationship $p_{i j}^{\mathrm{A}+\mathrm{B}}=g^{-1}\left[g\left(p_{i}^{\mathrm{A}}\right)+g\left(p_{j}^{\mathrm{B}}\right)\right]$ for a function $g$, can make the Tsallis entropy additive, and thus, recover its extensivity (e.g., Tsallis et al., 2005; Ruseckas, 2015). (Note that in his book, Tsallis (2009) goes to large lengths to show that it is possible to find systems for which the BG entropy is not extensive. On the other hand, he argues that there are certain systems for which the entropic form can be extensive, for certain values of the entropic index $q$. In fact, he mentions in the preface that the term "nonextensive entropy" is somewhat incorrect in this sense, but that it stuck for historical reasons.)

The two statistical formalisms, classical BG and Tsallis nonextensive, have the common property that their entropy becomes additive for a specific function $g$ in the relationship $p_{i j}^{\mathrm{A}+\mathrm{B}}=g^{-1}\left[g\left(p_{i}^{\mathrm{A}}\right)+g\left(p_{j}^{\mathrm{B}}\right)\right]$, that is, $g(x) \propto \ln (x)$ and $g(x) \propto\left(x^{q-1}-1\right) /(q-1)$, respectively (the latter is related to the $q$ deformed logarithm; see Silva et al., 1998; Yamano, 2002).

It is important that the above probability relationship is a characteristic feature of the canonical probability distribution in both the formalisms. In other words, the probability distribution that maximizes the BG entropy under the constraints of the canonical ensemble conforms to the correlations expressed by $g(x) \propto \ln (x)$ or $p_{i j}^{\mathrm{A}+\mathrm{B}}=p_{i}^{\mathrm{A}} \cdot p_{j}^{\mathrm{B}}$, which simply means zero correlation (due to the factorization of the exponentials; Livadiotis and McComas, 2011b) that makes the entropy additive. Also, the probability distribution that maximizes the Tsallis entropy under the same constraints 
(i.e., $q$ exponential or kappa distribution) conforms to the specific correlations expressed by $g(x) \propto\left(x^{q-1}-1\right) /(q-1)$ or $\left(p_{i j}^{\mathrm{A}+\mathrm{B}}\right)^{q-1}=\left(p_{i}^{\mathrm{A}}\right)^{q-1}+\left(p_{j}^{\mathrm{B}}\right)^{q-1}-1$, which again makes the entropy additive. In Sect. 3 we show this similar property of the two statistical formalisms in detail.

Then, we may ask the following question: is the property of BG and Tsallis entropies described above a general feature of any physically meaningful entropic function? Or can we reverse the question, and ask which specific entropic function follows the above properties? It will be really intriguing if we can determine the entropic formula appropriate for describing the plasma particle populations, simply by setting the following two first principles' properties: (1) additive energy, (2) additive entropy; i.e., the probability distribution derived by maximizing the entropy under the constraints of the canonical ensemble makes the entropy additive. This will be the main purpose of this paper and will be examined in Sect. 4.

\section{Canonical ensemble distributions with additive energy lead to additive entropy}

\subsection{The Gibbs' path}

The Gibbs' path (1902) for the maximization of the entropy $S\left(p_{1}, p_{2}, \ldots, p_{W}\right)$ under the constraints of a canonical ensemble (i.e., (i) normalization $1=\sum_{k=1}^{W} p_{k}$ and (ii) fixed internal energy $U=\sum_{k=1}^{W} p_{k} \varepsilon_{k}$ ) involves maximizing the functional

$$
\begin{aligned}
G\left(p_{1}, p_{2}, \ldots, p_{W}\right) & =S\left(p_{1}, p_{2}, \ldots, p_{W}\right)+\lambda_{1} \sum_{k=1}^{W} p_{k} \\
& +\lambda_{2} \sum_{k=1}^{W} p_{k} \varepsilon_{k} .
\end{aligned}
$$

Next, we examine the BG and Tsallis entropic formulations.

\subsection{BG entropy}

First, we start from the classical case of BG entropy

$S\left(p_{1}, p_{2}, \ldots, p_{W}\right)=-\sum_{k=1}^{W} p_{k} \ln \left(p_{k}\right)$,

where we ignored the Boltzmann constant $k_{\mathrm{B}}$ for simplicity. Then, setting $\left(\partial / \partial p_{j}\right) G\left(p_{1}, p_{2}, \ldots, p_{W}\right)=0$ to

$$
\begin{aligned}
G\left(p_{1}, p_{2}, \ldots, p_{W}\right)= & -\sum_{k=1}^{W} p_{k} \ln \left(p_{k}\right)+\lambda_{1} \sum_{k=1}^{W} p_{k} \\
& +\lambda_{2} \sum_{k=1}^{W} p_{k} \varepsilon_{k},
\end{aligned}
$$

we find

$p_{j}\left(\varepsilon_{j}\right)=\exp \left(\lambda_{1}-1\right) \cdot \exp \left(\lambda_{2} \varepsilon_{j}\right)$.

We may write Eq. (4) in a logarithmic form, $\ln p_{j}=\lambda_{2} \varepsilon_{j}+$ $\lambda_{1}-1$. Then, we separate the particle system into two parts, $A$ and B, so that each part is a new subsystem for which Eq. (4) holds:

$\ln p_{i}^{\mathrm{A}}=\lambda_{2} \varepsilon_{i}^{\mathrm{A}}+\lambda_{1}-1 \quad$ and $\quad \ln p_{j}^{\mathrm{B}}=\lambda_{2} \varepsilon_{j}^{\mathrm{B}}+\lambda_{1}-1$

The whole system is characterized by the joint probability, $p_{i j}^{\mathrm{A}+\mathrm{B}}$, meaning the probability of a particle in the subsystem A of residing in the state $i$ and a particle in the subsystem $\mathrm{B}$ of residing in the state $j$. This is related with the energy $\varepsilon_{i j}^{\mathrm{A}+\mathrm{B}}$ of the two-particle state,

$\ln p_{i j}^{\mathrm{A}+\mathrm{B}}=\lambda_{2} \varepsilon_{i j}^{\mathrm{A}+\mathrm{B}}+\lambda_{1}-1$.

Trivially, the energy of the two-particle state energy $\varepsilon_{i j}^{\mathrm{A}+\mathrm{B}}$ equals the summation of the energy of each particle (since no interparticle force is considered); i.e., the system's energy is additive:

$\varepsilon_{i j}^{\mathrm{A}+\mathrm{B}}=\varepsilon_{i}^{\mathrm{A}}+\varepsilon_{j}^{\mathrm{B}}$.

Hence, by eliminating energies from Eqs. (5) and (6), we find

$$
\begin{aligned}
& \ln p_{i j}^{\mathrm{A}+\mathrm{B}}+\left(\lambda_{1}-1\right)=\lambda_{2} \varepsilon_{i}^{\mathrm{A}}+\left(\lambda_{1}-1\right)+\lambda_{2} \varepsilon_{j}^{\mathrm{B}}+\left(\lambda_{1}-1\right) \\
& =\ln p_{i}^{\mathrm{A}}+\ln p_{j}^{\mathrm{B}} \quad \text { or } \\
& p_{i j}^{\mathrm{A}+\mathrm{B}}=p_{i}^{\mathrm{A}} \cdot p_{j}^{\mathrm{B}} \cdot e^{-\left(\lambda_{1}-1\right)} \text {. }
\end{aligned}
$$

At this point we recall that the Lagrange multipliers, $\lambda_{1}$ and $\lambda_{2}$, are related with the partition function $Z=e^{-\left(\lambda_{1}-1\right)}$ and the inverse temperature $\beta=-\lambda_{2}$, respectively, and they are not necessarily equal for the two subsystems A and B, or the whole system $A+B$. Nevertheless, the logarithm of the partition function or $\left(\lambda_{1}-1\right)$ is an extensive parameter, i.e., $\left(\lambda_{1}-1\right)^{\mathrm{A}+\mathrm{B}}=\left(\lambda_{1}-1\right)^{\mathrm{A}}+\left(\lambda_{1}-1\right)^{\mathrm{B}}$, while the temperature is not an extensive parameter and can be considered the same: $\lambda_{2}^{\mathrm{A}+\mathrm{B}}=\lambda_{2}^{\mathrm{A}}=\lambda_{2}^{\mathrm{B}}$. Then, instead of Eqs. (8) and (9), we obtain

$$
\begin{aligned}
\ln p_{i j}^{\mathrm{A}+\mathrm{B}} & =\lambda_{2} \varepsilon_{i j}^{\mathrm{A}+\mathrm{B}}+\left(\lambda_{1}-1\right)^{\mathrm{A}+\mathrm{B}} \\
& =\lambda_{2} \varepsilon_{i}^{\mathrm{A}}+\left(\lambda_{1}-1\right)^{\mathrm{A}}+\lambda_{2} \varepsilon_{j}^{\mathrm{B}}+\left(\lambda_{1}-1\right)^{\mathrm{B}} \\
& =\ln p_{i}^{\mathrm{A}}+\ln p_{j}^{\mathrm{B}},
\end{aligned}
$$

which clearly shows that the canonical probabilities are independent:

$\ln \left(p_{i j}^{\mathrm{A}+\mathrm{B}}\right)=\ln \left(p_{i}^{\mathrm{A}}\right)+\ln \left(p_{j}^{\mathrm{B}}\right) \Rightarrow p_{i j}^{\mathrm{A}+\mathrm{B}}=p_{i}^{\mathrm{A}} \cdot p_{j}^{\mathrm{B}}$.

Equation (9) indicates that the result in Eq. (11) can be obtained simply by setting $\lambda_{1}=1$. Certainly, this restricts the generality, but it can be used as a trick to simplify the calculations. Furthermore, we can easily obtain the additivity of entropy. Indeed, applying the operator $\sum_{i=1}^{W} \sum_{j=1}^{W} p_{i j}^{\mathrm{A}+\mathrm{B}} \times$ to 
both sides of Eq. (11), we obtain

$$
\begin{aligned}
p_{i j}^{\mathrm{A}+\mathrm{B}} \ln p_{i j}^{\mathrm{A}+\mathrm{B}}=p_{i j}^{\mathrm{A}+\mathrm{B}} \ln p_{i}^{\mathrm{A}} & +p_{i j}^{\mathrm{A}+\mathrm{B}} \ln p_{j}^{\mathrm{B}} \\
\Rightarrow-\sum_{i=1}^{W} \sum_{j=1}^{W} p_{i j}^{\mathrm{A}+\mathrm{B}} \ln p_{i j}^{\mathrm{A}+\mathrm{B}}= & -\sum_{i=1}^{W} p_{i}^{\mathrm{A}} \ln \left(p_{i}^{\mathrm{A}}\right) \\
& -\sum_{j=1}^{W} p_{j}^{\mathrm{B}} \ln \left(p_{j}^{\mathrm{B}}\right)
\end{aligned}
$$

because $\sum_{j=1}^{W} p_{i j}^{\mathrm{A}+\mathrm{B}}=p_{i}^{\mathrm{A}}$ and $\sum_{i=1}^{W} p_{i j}^{\mathrm{A}+\mathrm{B}}=p_{j}^{\mathrm{B}}$. Hence, we arrive at the additivity of the entropy of the system to the entropies of the subsystems,

$S^{\mathrm{A}+\mathrm{B}}=S^{\mathrm{A}}+S^{\mathrm{B}}$.

\subsection{Tsallis entropy}

Next, we continue with the Tsallis $q$ entropy,

$$
\begin{aligned}
S\left(p_{1}, p_{2}, \ldots, p_{W}\right) & =\frac{1-\varphi\left(p_{1}, p_{2}, \ldots, p_{W}\right)}{q-1} \\
& =\frac{1}{q-1} \cdot \sum_{k=1}^{W}\left(p_{k}-p_{k}^{q}\right),
\end{aligned}
$$

(e.g., Havrda and Charvát, 1967; Tsallis, 1988) where the $\operatorname{argument} \varphi$ is defined by

$\varphi\left(p_{1}, p_{2}, \ldots, p_{W}\right)=\sum_{k=1}^{W} p_{k}^{q}$.

Again, the maximization of the entropy under the constraints of the canonical ensemble involves maximizing the functional

$$
\begin{aligned}
G\left(p_{1}, p_{2}, \ldots, p_{W}\right) & =\frac{1}{q-1} \cdot \sum_{k=1}^{W}\left(p_{k}-p_{k}^{q}\right)+\lambda_{1} \sum_{k=1}^{W} p_{k} \\
& +\lambda_{2} \sum_{k=1}^{W} p_{k} \varepsilon_{k} .
\end{aligned}
$$

Note that for simplicity we do not use the formulation of escort distributions (Beck and Schlogl, 1993). The dyadic formalism of ordinary/escort distributions is of fundamental importance in modern nonextensive statistical mechanics (Livadiotis, 2017a; chap. 1). It has been shown that this dyadic formalism of distributions can be avoided in order to simplify the theory, but it leads to a dyadic formulation of entropy (Livadiotis, 2017b).

Hence, $\left(\partial / \partial p_{j}\right) G\left(p_{1}, p_{2}, \ldots, p_{W}\right)=0$ gives

$$
\begin{aligned}
p_{j}\left(\varepsilon_{j}\right)= & {\left[1+\left(1-q^{-1}\right) \cdot\left(\lambda_{1}-1\right)\right]^{\frac{q^{-1}}{1-q^{-1}}} } \\
& \times\left[1+\left(1-q^{-1}\right) \cdot \frac{\lambda_{2} \varepsilon_{j}}{1+\left(1-q^{-1}\right) \cdot\left(\lambda_{1}-1\right)}\right]^{\frac{q^{-1}}{1-q^{-1}}}
\end{aligned}
$$

or

$$
p_{j}\left(\varepsilon_{j}\right)=\exp _{q^{-1}}^{q^{-1}}\left(\lambda_{1}-1\right) \cdot \exp _{q^{-1}}^{q^{-1}}\left[\frac{\lambda_{2} \varepsilon_{j}}{1_{q^{-1}}\left(\lambda_{1}-1\right)}\right],
$$

which reflects a generalization of Eq. (4). We used the $Q$-deformed exponential function, and its inverse, the $Q$ logarithm function (Silva et al., 1998; Yamano, 2002), defined by

$\exp _{Q}(x)=[1+(1-Q) \cdot x]_{+}^{-\frac{1}{Q-1}}$

$\ln _{Q}(x)=\frac{1-x^{1-Q}}{Q-1}$.

We also used the $Q$-deformed "unity function" (Livadiotis and McComas, 2009), defined by

$1_{Q}(x)=[1+(1-Q) \cdot x]_{+}$.

The subscript "+" in $[\ldots]_{+}$denotes the cut-off condition, where $\exp _{Q}(x)$ becomes zero if its base [...] is non-positive. Therefore, Eq. (17b) leads to

$$
\begin{aligned}
& p_{j}^{q-1}=1+\left(1-q^{-1}\right) \cdot\left(\lambda_{1}-1\right)+\left(1-q^{-1}\right) \cdot \lambda_{2} \varepsilon_{j}, \\
& \frac{1-p_{j}^{q-1}}{q^{-1}-1}=\ln _{q^{-1}}\left(p_{j}^{q}\right)=-q \cdot \ln _{q}\left(p_{j}^{-1}\right) \\
& =\lambda_{2} \varepsilon_{j}+\left(\lambda_{1}-1\right)
\end{aligned}
$$

Dividing again the whole system into two subsystems, A and $\mathrm{B}$, using the additivity of energy, and setting $\lambda_{1}=1$, the independence relation (Eq. 11) is generalized to

$$
\begin{aligned}
& \ln _{q}\left[\left(p_{i j}^{\mathrm{A}+\mathrm{B}}\right)^{-1}\right]=\ln _{q}\left[\left(p_{i}^{\mathrm{A}}\right)^{-1}\right]+\ln _{q}\left[\left(p_{j}^{\mathrm{B}}\right)^{-1}\right] \\
& \Rightarrow\left(p_{i j}^{\mathrm{A}+\mathrm{B}}\right)^{q-1}=\left(p_{i}^{\mathrm{A}}\right)^{q-1}+\left(p_{j}^{\mathrm{B}}\right)^{q-1}-1,
\end{aligned}
$$

which is sometimes called the $q$ independence relationship (Umarov et al., 2008). Then, we apply the operator $\sum_{i=1}^{W} \sum_{j=1}^{W} p_{i j}^{\mathrm{A}+\mathrm{B}} \times$,

$$
\begin{aligned}
\sum_{i=1}^{W} \sum_{j=1}^{W}\left(p_{i j}^{\mathrm{A}+\mathrm{B}}\right)^{q} & =\sum_{i=1}^{W}\left(p_{i}^{\mathrm{A}}\right)^{q}+\sum_{j=1}^{W}\left(p_{j}^{\mathrm{B}}\right)^{q} \\
-1 \Rightarrow \varphi^{\mathrm{A}+\mathrm{B}}=\varphi^{\mathrm{A}}+\varphi^{\mathrm{B}}-1 &
\end{aligned}
$$

and using the entropic formula (Eq. 14), we end up with the additivity of entropy, as shown in Eq. (13).

Note that the additivity leads to the extensivity: the additivity for a function $f$ is expressed by $f(\mathrm{~A}+\mathrm{B})=f(\mathrm{~A})+f(\mathrm{~B})$, or considering $N$ different subsystems,

$$
f\left(\bigcup_{n=1}^{N} \mathrm{~A}_{n}\right)=\sum_{n=1}^{N} f\left(\mathrm{~A}_{n}\right)
$$

while the extensivity is expressed by

$$
f\left(\bigcup_{n=1}^{N} \mathrm{~A}_{0}\right)=N \cdot f\left(\mathrm{~A}_{0}\right) .
$$


Therefore, the canonical probability distribution, the one that maximizes the entropy under the constraints of a canonical ensemble, makes the entropy additive (and therefore extensive) if the energy is additive. Several special conditions can simplify this result, e.g., constant Lagrange constraints with $\lambda_{1}=1$ (i.e., independent of the probability distribution). This is, however, the case for both the entropic formulation of classical BG and Tsallis nonextensive statistical mechanics.

Next, we reverse the problem and seek to find the specific entropic formula for which both the energy and entropy are additive.

\section{Additive energy and entropy leads to Tsallis entropic formalism}

The general entropic form is still function of the probabilities: $S=S\left(\left\{p_{k}\right\}_{k=1}^{W}\right)$. Then, its derivative with respect to any of the probability components, for example the $i$ th, is also a function of all of these components, i.e., $\partial S / \partial p_{i}=$ $F_{i}\left(\left\{p_{k}\right\}_{k=1}^{W}\right)$, for any $i: 1, \ldots, W$. However, the second constraint (i.e., fixed internal energy) of the canonical ensemble connects the $i$ th entropic derivative to a function $h_{i}$ of the $i$ th energy, $\varepsilon_{i}$, namely, $\partial S / \partial p_{i}=h_{i}\left(\varepsilon_{i}\right)$. On the other hand, the canonical probability distribution derived from the entropy maximization constitutes an expression of the $i$ th probability component with some invertible function $g$ of the $i$ th energy, $p_{i}=g\left(\varepsilon_{i}\right)$. Therefore, we conclude that $\partial S / \partial p_{i}=$ $F_{i}\left(p_{i}\right)$, where $F_{i}=h_{i} \circ g^{-1}$; in other words, the entropy can be factorized as a summation of functions of each probability component, $S=\sum_{k=1}^{W} f_{k}\left(p_{k}\right)$, where we set $f_{i}\left(p_{i}\right)=$ $\int F_{i}\left(p_{i}\right) d p_{i}$. Finally, we consider that none of the states $(k=1, \ldots, W)$ should have a special effect on the entropy; i.e., each state "weights" the same, so the entropic functional $S=S\left(\left\{p_{k}\right\}_{k=1}^{W}\right)$ should be symmetric to any permutation of each components, e.g., $S=S\left(\ldots, p_{k}, \ldots, p_{\ell}, \ldots\right)=$ $S\left(\ldots, p_{\ell}, \ldots, p_{k}, \ldots\right)$ (i.e., the entropy is invariant under any relabeling of the states). This leads to $f_{k}=f$; hence, considering (1) entropy maximization and (2) no weighting, we obtain

$S=\sum_{k=1}^{W} f\left(p_{k}\right)$

For example, in the cases of Boltzmann (Eq. 2) and Tsallis (Eq. 14) entropies, the function $f$ is respectively given by

$f(x)=-x \ln (x)$ and $f(x)=\left(x-x^{q}\right) /(q-1)$.

The maximization of entropy under the constraints of a canonical ensemble, i.e., $1=\sum_{k=1}^{W} p_{k}$ and $U=\sum_{k=1}^{W} p_{k} \varepsilon_{k}$, involves maximizing the functional $G\left(\left\{p_{k}\right\}_{k=1}^{W}\right)=\sum_{k=1}^{W} f\left(p_{k}\right)+\lambda_{1} \sum_{k=1}^{W} p_{k}+\lambda_{2} \sum_{k=1}^{W} p_{k} \varepsilon_{k}$.
Hence, setting $\partial G\left(\left\{p_{k}\right\}_{k=1}^{W}\right) / \partial p_{i}=0$, we obtain

$F\left(p_{i}\right)+\lambda_{1}+\lambda_{2} \varepsilon_{i}=0, \quad$ or $\quad p_{i}\left(\varepsilon_{i}\right)=F^{-1}\left(-\lambda_{1}-\lambda_{2} \varepsilon_{i}\right)$,

with $F(x) \equiv f^{\prime}(x)$.

We now consider two systems $\mathrm{A}$ and $\mathrm{B}$, with respective energy spectra $\left\{\varepsilon_{i}^{\mathrm{A}}\right\}_{i=1}^{W}$ and $\left\{\varepsilon_{j}^{\mathrm{B}}\right\}_{j=1}^{W}$, associated with the discrete probability distributions $\left\{p_{i}^{\mathrm{A}}\right\}_{i=1}^{W}$ and $\left\{p_{j}^{\mathrm{B}}\right\}_{j=1}^{W}$. The total system $\mathrm{A}+\mathrm{B}$ has an energy spectrum $\left\{\varepsilon_{i j}^{\mathrm{A}+\mathrm{B}}\right\}_{i, j=1}^{W}$, associated with the joint probability distribution $\left\{p_{i j}^{\mathrm{A}+\mathrm{B}}\right\}_{i, j=1}^{W}$. The probability distributions $\left\{p_{i}^{\mathrm{A}}\right\}_{i=1}^{W}$ and $\left\{p_{j}^{\mathrm{B}}\right\}_{j=1}^{W}$ are marginal of the joint distribution, i.e., $\sum_{j=1}^{W} p_{i j}^{\mathrm{A}+\mathrm{B}}=p_{i}^{\mathrm{A}}$ and $\sum_{i=1}^{W} p_{i j}^{\mathrm{A}+\mathrm{B}}=p_{j}^{\mathrm{B}}$. As we will find further below, the joint probability can be expressed as a function of the marginal probabilities, $p_{i j}^{\mathrm{A}+\mathrm{B}}=H\left(p_{i}^{\mathrm{A}}, p_{j}^{\mathrm{B}}\right)$. On the other hand, the relation between the joint energies $\varepsilon_{i j}^{\mathrm{A}+\mathrm{B}}$ is rather trivial to be derived: particles in $\mathrm{A}$ with energy $\varepsilon_{i}^{\mathrm{A}}$ and particles in $\mathrm{B}$ with energy $\varepsilon_{j}^{\mathrm{B}}$ ensemble the particles in $\mathrm{A}+\mathrm{B}$ with energy $\varepsilon_{i j}^{\mathrm{A}+\mathrm{B}}=\varepsilon_{i}^{\mathrm{A}}+\varepsilon_{j}^{\mathrm{B}}$. Trivially, the same additivity holds for their mean values - the internal energies:

$$
\begin{aligned}
U^{\mathrm{A}+\mathrm{B}} & =\sum_{i, j} p_{i j}^{\mathrm{A}+\mathrm{B}} \varepsilon_{i j}^{\mathrm{A}+\mathrm{B}}=\sum_{i, j} p_{i j}^{\mathrm{A}+\mathrm{B}} \varepsilon_{i}^{\mathrm{A}}+\sum_{i, j} p_{i j}^{\mathrm{A}+\mathrm{B}} \varepsilon_{j}^{\mathrm{B}} \\
& =\sum_{i} p_{i}^{\mathrm{A}} \varepsilon_{i}^{\mathrm{A}}+\sum_{j} p_{j}^{\mathrm{B}} \varepsilon_{j}^{\mathrm{B}}=U^{\mathrm{A}}+U^{\mathrm{B}} .
\end{aligned}
$$

Now, the probability distributions are related to their energies, according to Eq. (7). According to Eq. (25), we have

$$
\begin{aligned}
& F\left(p_{i}^{\mathrm{A}}\right)+\lambda_{1}+\lambda_{2} \varepsilon_{i}^{\mathrm{A}}=0, \quad F\left(p_{j}^{\mathrm{B}}\right)+\lambda_{1}+\lambda_{2} \varepsilon_{j}^{\mathrm{B}}=0, \\
& F\left(p_{i j}^{\mathrm{A}+\mathrm{B}}\right)+\lambda_{1}+\lambda_{2} \varepsilon_{i j}^{\mathrm{A}+\mathrm{B}}=0,
\end{aligned}
$$

and due to the additivity of energies, we obtain

$$
F\left(p_{i j}^{\mathrm{A}+\mathrm{B}}\right)-\lambda_{1}=F\left(p_{i}^{\mathrm{A}}\right)+F\left(p_{j}^{\mathrm{B}}\right) .
$$

Again, the Lagrange constants, $\lambda_{1}$ and $\lambda_{2}$, are considered to be independent of the probability distribution. Setting $\widetilde{F} \equiv$ 
$\frac{1}{-\lambda_{1}} F$, Eq. (28) becomes

$$
\begin{aligned}
& {\left[\widetilde{F}\left(p_{i j}^{\mathrm{A}+\mathrm{B}}\right)-1\right]=\left[\widetilde{F}\left(p_{i}^{\mathrm{A}}\right)-1\right]+\left[\widetilde{F}\left(p_{j}^{\mathrm{B}}\right)-1\right],} \\
& p_{i j}^{\mathrm{A}+\mathrm{B}}=H\left(p_{i}^{\mathrm{A}}, p_{j}^{\mathrm{B}}\right) \\
& \quad \text { with } \quad H(x, y) \equiv \widetilde{F}^{-1}[\widetilde{F}(x)+\widetilde{F}(y)-1] .
\end{aligned}
$$

Then, we apply $\sum_{i=1}^{W} \sum_{j=1}^{W} p_{i j}^{\mathrm{A}+\mathrm{B}} \times$ to both sides of Eq. (29):

$$
\begin{gathered}
\sum_{i, j=1}^{W}\left[\widetilde{F}\left(p_{i j}^{\mathrm{A}+\mathrm{B}}\right)-1\right] p_{i j}^{\mathrm{A}+\mathrm{B}}=\sum_{i=1}^{W}\left[\widetilde{F}\left(p_{i}^{\mathrm{A}}\right)-1\right] \sum_{j=1}^{W} p_{i j}^{\mathrm{A}+\mathrm{B}} \\
+\sum_{j=1}^{W}\left[\widetilde{F}\left(p_{j}^{\mathrm{B}}\right)-1\right] \sum_{i=1}^{W} p_{i j}^{\mathrm{A}+\mathrm{B}}, \quad \text { or } \\
\sum_{i, j}^{W}\left[\widetilde{F}\left(p_{i j}^{\mathrm{A}+\mathrm{B}}\right)-1\right] p_{i j}^{\mathrm{A}+\mathrm{B}}=\sum_{i}^{W}\left[\widetilde{F}\left(p_{i}^{\mathrm{A}}\right)-1\right] p_{i}^{\mathrm{A}} \\
+\sum_{j}^{W}\left[\widetilde{F}\left(p_{j}^{\mathrm{B}}\right)-1\right] p_{j}^{\mathrm{B}} .
\end{gathered}
$$

(Note that the number of states allowed may be different for the two subsystems, $W_{A} \neq W_{B}$, but here it does not make any difference to consider $W_{A}=W_{B}=W$.)

We recall that $\widetilde{F}(x) \equiv \frac{1}{-\lambda_{1}} f^{\prime}(x)$; thus, we find

$$
\begin{aligned}
& \sum_{i, j}^{W}\left[\frac{1}{-\lambda_{1}} f^{\prime}\left(p_{i j}^{\mathrm{A}+\mathrm{B}}\right)-1\right] p_{i j}^{\mathrm{A}+\mathrm{B}}= \\
& \sum_{i}^{W}\left[\frac{1}{-\lambda_{1}} f^{\prime}\left(p_{i}^{\mathrm{A}}\right)-1\right] p_{i}^{\mathrm{A}}+\sum_{j}^{W}\left[\frac{1}{-\lambda_{1}} f^{\prime}\left(p_{j}^{\mathrm{B}}\right)-1\right] p_{j}^{\mathrm{B}} .
\end{aligned}
$$

We compare this relationship with the additivity of entropy:

$$
\begin{aligned}
S^{\mathrm{A}+\mathrm{B}} & =\sum_{i, j}^{W} f\left(p_{i j}^{\mathrm{A}+\mathrm{B}}\right)=\sum_{i}^{W} f\left(p_{i}^{\mathrm{A}}\right)+\sum_{j}^{W} f\left(p_{j}^{\mathrm{B}}\right) \\
& =S^{\mathrm{A}}+S^{\mathrm{B}} .
\end{aligned}
$$

The two functions $f(x)$ and $\left[\frac{1}{-\lambda_{1}} f^{\prime}(x)-1\right] \cdot x$ have the same additivity property. Therefore, one function $f$ that can ensure the additivity of entropy is the one that obeys conforms to the proportionality, $f(x) \propto\left[\frac{1}{-\lambda_{1}} f^{\prime}(x)-1\right] \cdot x$, or the differential equation

$$
\begin{aligned}
& f(x)=c \cdot\left[\frac{1}{-\lambda_{1}} f^{\prime}(x)-1\right] \cdot x, \\
& \text { or } f^{\prime}(x)+\frac{\lambda_{1}}{c} \frac{1}{x} f(x)=-\lambda_{1},
\end{aligned}
$$

with solution

$f(x)=\lambda_{1} \cdot \frac{x-x^{\frac{-\lambda_{1}}{c}}}{\frac{-\lambda_{1}}{c}-1}+f(1) \cdot x^{\frac{-\lambda_{1}}{c}}$.
(Note that the selection of proportionality between the two functions $f(x)$ and $\left[\frac{1}{-\lambda_{1}} f^{\prime}(x)-1\right] \cdot x$ makes the derivation of Eq. (34) a sufficient but not necessary condition. Other functional forms may also exist, for example, a linear combination of the two functions mentioned.)

A fully organized system has zero entropy, so that $S\left(p_{i}=1, p_{j}=0 \forall j: 1, \ldots, W\right.$, with $\left.j \neq i\right)=0$. Then, from Eq. (24a) we find $S=0=f(1)+(W-1) f(0)$. Equation (35) gives $f(0)=0$; hence, we find $f(1)=0$, too. Then, we set $q \equiv \frac{-\lambda_{1}}{c}$, where we find

$f(x)=\lambda_{1} \cdot \frac{x-x^{q}}{q-1}$,

or, setting also $\lambda_{1}=1$ (that is, setting the entropic unit $k_{B}$ equal to 1 ), we end up with

$f(x)=\frac{x-x^{q}}{q-1}$.

Therefore, the entropic function $S=\sum_{k=1}^{W} f\left(p_{k}\right)$ becomes

$S=\frac{1}{q-1} \cdot \sum_{k=1}^{W}\left(p_{k}-p_{k}^{q}\right)$,

that is, the Tsallis entropic formulation that builds the nonextensive statistical mechanics.

We note that Eq. (33) is invariant under linear transformations:

$$
\begin{aligned}
f(x) \rightarrow f(x)+a(x+b) \quad \text { with } \quad a & =\frac{\lambda_{1}-1}{1+(q-1) \frac{W-1}{W-2},} \\
b & =\frac{1}{W(W-2)},
\end{aligned}
$$

which leads again to Eq. (38).

\section{Conclusions}

The paper resolved a basic problem about the origin of the distributions and statistical mechanics applied in space plasmas. Kappa distributions, or combinations/superposition thereof, can describe the velocities and energies of the plasma populations in space plasmas. While these empirical distributions have been used since the mid-1960s for modeling space plasma datasets, their statistical origin remained unknown. It was just about a decade ago that the connection of these distributions with the statistical framework of nonextensive statistical mechanics was completed and understood (Livadiotis, 2017a; chap. 1). Indeed, the kappa distribution is the outcome of the maximization of the $q$ entropy of Tsallis under the constraints of a canonical ensemble (identifying the $q$ exponential distributions, first used in a statistical framework context in Tsallis, 1988, as kappa distributions). Once this concept was understood by the science community, 
the next question was about the physical origin and reasoning of this entropic formula. This paper showed that the $q$ entropy, which is the entropic formula that when maximized leads to the kappa distribution, can be derived under simple first principles and conditions, namely, by considering that energy and entropy are both additive physical quantities.

Competing interests. The authors declare that they have no conflict of interest.

Special issue statement. This article is part of the special issue "Nonlinear Waves and Chaos". It is a result of the 10th International Nonlinear Wave and Chaos Workshop (NWCW17), San Diego, United States, 20-24 March 2017.

Acknowledgements. The work was supported in part by the project NNX17AB74G of NASA's HGI Program.

Edited by: Yasuhito Narita

Reviewed by: three anonymous referees

\section{References}

Abe, S.: Axioms and uniqueness theorem for Tsallis entropy, Phys. Lett. A, 271, 74-79, 2000.

Abe, S. and Suzuki, N.: Itineration of the Internet over nonequilibrium stationary states in Tsallis statistics, Phys. Rev. E, 67, 016106, , doi: https://doi.org/10.1103/PhysRevE.67.016106, 2003.

Andricioaei, I. and Straub, J. E.: Generalized simulated annealing algorithms using Tsallis statistics: application to conformational optimization of a tetrapeptide, Phys. Rev. E, 53, R3055-R3058, 1996.

Baluku, T. K., Hellberg, M. A., Kourakis, I., and Saini, N. S.: Dust ion acoustic solitons in a plasma with kappadistributed electrons, Phys. Plasmas, 17, 053702, https://doi.org/10.1063/1.3400229, 2010.

Beck, C. and Schlögl, F.: Thermodynamics of Chaotic Systems, Cambridge University Press, Cambridge, 1993.

Bian, N., Emslie, G. A., Stackhouse, D. J., and Kontar, E. P.: The formation of a kappa-distribution accelerated electron populations in solar flares, Astrophys. J., 796, 142, https://doi.org/10.1088/0004-637X/796/2/142, 2014.

Binsack, J. H.: Plasma studies with the IMP-2 satellite, Ph.D. thesis, MIT, Massachusetts Institute of Technology, 1966.

Borges, E. P., Tsallis, C., Anãnõs, G. F. J., and De Oliveira, P. M. C.: Nonequilibrium probabilistic dynamics at the logistic map edge of chaos, Phys. Rev. Lett., 89, 254103, https://doi.org/10.1103/PhysRevLett.89.254103, 2002.

Borland, L.: Option pricing formulas based on a nonGaussian stock price model, Phys. Rev. Lett., 89, 098701, https://doi.org/10.1103/PhysRevLett.89.098701, 2002.

Broiles, T. W., Livadiotis, G., Burch, J. L., Chae, K., Clark, G., Cravens, T. E., Davidson, R., Eriksson, A., Frahm, R. A., Fuse- lier, S. A., Goldstein, J., Goldstein, R., Henri, P., Madanian, H., Mandt, K. E., Mokashi, P., Pollock, C., Rahmati, A., Samara, M., Schwartz, S. J.: Characterizing cometary electrons with kappa distributions, J. Geophys. Res., 121, 7407-7422, 2016 a.

Broiles, T. W., Burch, J. L., Chae, K., Clark, G., Cravens, T. E., Eriksson, A., Fuselier, S. A., Frahm, R. A., Gasc, S., Goldstein, R., Henri, P., Koenders, C., Livadiotis, G., Mandt, K. E., Mokashi, P., Nemeth, Z., Rubin, M., and Samara, M.: Statistical analysis of suprathermal electron drivers at 67P/ChuryumovGerasimenko, Mon. Not. R. Astron. Soc., 462, S312-S322, 2016b.

Bryant, D. A.: Debye length in a kappa-distribution, J. Plasma Phys., 56, 87-93, https://doi.org/10.1017/S0022377800019115, 1996.

Carbary, J. F., Kane, M., Mauk, B. H., and Krimigis, S. M.: Using the kappa function to investigate hot plasma in the magnetospheres of the giant planets, J. Geophys. Res., 119, 8426-8447, 2014.

Chotoo, K., Schwadron, N. A., Mason, G. M., Zurbuchen, T. H., Gloeckler, G., Posner, A., Fisk, L. A., Galvin, A. B., Hamilton, D. C., and Collier, M. R.: The suprathermal seed population for corotaing interaction region ions at $1 \mathrm{AU}$ deduced from composition and spectra of $\mathrm{H}^{+}, \mathrm{He}^{++}$, and $\mathrm{He}^{+}$observed by Wind, J. Geophys. Res., 105, 23107-23122, 2000.

Christon, S. P.: A comparison of the Mercury and earth magnetospheres: electron measurements and substorm time scales, Icarus, 71, 448-471, 1987.

Collier, M. R. and Hamilton, D. C.: The relationship between kappa and temperature in the energetic ion spectra at Jupiter, Geophys. Res. Lett., 22, 303-306, 1995.

Cranmer, S. R.: Suprathermal electrons in the solar corona: can nonlocal transport explain heliospheric charge states?, Astrophys. J. Lett., 791, L31, https://doi.org/10.1088/2041-8205/791/2/L31, 2014.

Decker, R. B. and Krimigis, S. M.: Voyager observations of lowenergy ions during solar cycle 23, Adv. Space Res., 32, 597-602, 2003.

Decker, R. B., Krimigis, S. M., Roelof, E. C., Hill, M. E., Armstrong, T. P., Gloeckler, G., Hamilton, D. C., and Lanzerotti, L. J.: Voyager 1 in the foreshock, termination shock, and heliosheath, Science, 309, 2020-2024, 2005.

Dialynas, K., Krimigis, S. M., Mitchell, D. G., Hamilton, D. C., Krupp, N., and Brandt, P. C.: Energetic ion spectral characteristics in the Saturnian magnetosphere using Cassini/MIMI measurements, J. Geophys. Res., 114, A01212, https://doi.org/10.1029/2008JA013761, 2009.

Dos Santos, M. S., Ziebell, L. F., and Gaelzer, R.: Ion firehose instability in a dusty plasma considering product-bi-kappa distributions for the plasma particles, Phys. Plasmas, 23, 013705, https://doi.org/10.1063/1.4939885, 2016.

Dos Santos, R. J. V.: Generalization of Shannon's theorem for Tsallis entropy, J. Math. Phys., 38, 4104-4107, 1997.

$\mathrm{Du}, \mathrm{J}$. : The nonextensive parameter and Tsallis distribution for selfgravitating systems, EPL-Europhys. Lett., 67, 893-899, 2004.

Dzifčáková, E. and Dudík, J.: H to Zn ionization equilibrium for the non-Maxwellian electron $\kappa$-distributions: updated calculations, Astrophys. J. Suppl. S., 206, https://doi.org/10.1088/00670049/206/1/6, 2013. 
Dzifčáková, E., Dudík, J., Kotrč, P., Fárník, F., and Zemanová, A.: KAPPA: a package for synthesis of optically thin spectra for the non-Maxwellian $\kappa$-distributions based on the Chianti database, Astrophys. J. Suppl. S., 217, https://doi.org/10.1088/00670049/217/1/14, 2015.

Eslami, P., Mottaghizadeh, M., and Pakzad, H. R.: Nonplanar dust acoustic solitary waves in dusty plasmas with ions and electrons following a q-nonextensive distribution, Phys. Plasmas, 18, 102303, https://doi.org/10.1063/1.3642639, 2011.

Fisk, L. A. and Gloeckler, G.: The case for a common spectrum of particles accelerated in the heliosphere: observations and theory, J. Geophys. Res., 119, 8733, 2014.

Formisano, V., Moreno, G., Palmiotto, F., and Hedgecock, P. C.: Solar wind interaction with the Earth's magnetic field 1. Magnetosheath, J. Geophys. Res., 78, 3714-3730, 1973.

Fuselier, S. A., Allegrini, F., Bzowski, M., Dayeh, M. A., Desai, M., Funsten, H. O., Galli, A., Heirtzler, D., Janzen, P., Kubiak, M. A., Kucharek, H., Lewis, W., Livadiotis, G., McComas, D. J., Möbius, E., Petrinec, S. M., Quinn, M., Schwadron, N., Sokół, J. M., Trattner, K. J., Wood, B. E., and Wurz, P.: Low energy neutral atoms from the heliosheath, Astrophys. J., 784, https://doi.org/10.1088/0004-637X/784/2/89, 2014.

Gibbs, J. W.: Elementary Principles in Statistical Mechanics, Scribner's sons, New York, 1902.

Gougam, L. A. and Tribeche, M.: Debye shielding in a nonextensive plasma, Phys. Plasmas, 18, 062102, https://doi.org/10.1063/1.3577599, 2011.

Grabbe, C.: Generation of broadband electrostatic waves in Earth's magnetotail, Phys. Rev. Lett., 84, 3614, https://doi.org/10.1103/PhysRevLett.84.3614, 2000.

Grassi, A.: A relationship between atomic correlation energy of neutral atoms and generalized entropy, Int. J. Quantum Chem., 111, 2390-2397, 2010.

Habeck, M., Nilges, M., and Rieping, W.: Replica-exchange Monte Carlo scheme for Bayesian data analysis, Phys. Rev. Lett., 94, 018105, https://doi.org/10.1103/PhysRevLett.94.018105, 2005.

Hapgood, M., Perry, C., Davies, J., and Denton, M.: The role of suprathermal particle measurements in CrossScale studies of collisionless plasma processes, Planet. Space Sci., 59, 618-629, 2011.

Havrda, J. and Charvát, F.: Concept of structural a-entropy, Kybernetika, 3, 30-35, 1967.

Heerikhuisen, J., Pogorelov, N. V., Florinski, V., Zank, G. P., and le Roux, J. A.: The effects of a k-distribution in the heliosheath on the global heliosphere and ENA flux at $1 \mathrm{AU}$, Astrophys. J., 682, 679-689, 2008.

Heerikhuisen, J., Zirnstein, E., and Pogorelov, N.: $\kappa$-distributed protons in the solar wind and their charge-exchange coupling to energetic hydrogen, J. Geophys. Res., 120, 1516-1525, 2015.

Hellberg, M. A., Mace, R. L., Baluku, T. K., Kourakis, I., and Saini, N. S.: Comment on "Mathematical and physical aspects of Kappa velocity distribution" [Phys. Plasmas 14, 110702 (2007)], Phys. Plasmas, 16, 094701, https://doi.org/10.1063/1.3213388, 2009.

Hou, S. Q., He, J. J., Parikh, A., Kahl, D., Bertulani, C. A., Kajino, T., Mathews, G. J., and Zhao, G.: Non-extensive statistics to the cosmological lithium problem, Astrophys. J., 834, 165, https://doi.org/10.3847/1538-4357/834/2/165, 2017.
Jeffrey, N. L. S., Fletcher, L., and Labrosse, N.: First evidence of non-Gaussian solar flare EUV spectral line profiles and accelerated non-thermal ion motion, Astron. Astrophys., 590, A99, https://doi.org/10.1051/0004-6361/201527986, 2016.

Jund, P., Kim, S. G., and Tsallis, C.: Crossover from extensive to nonextensive behavior driven by long-range interactions, Phys. Rev. B, 52, 50, https://doi.org/10.1103/PhysRevB.52.50, 1995.

Jurac, S., McGrath, M. A., Johnson, R. E., Richardson, J. D., Vasyliunas, V. M., and Eviatar, A.: Saturn: search for a missing water source, Geophys. Res. Lett., 29, 2172, https://doi.org/10.1029/2002GL015855, 2002.

Khinchin, A. I.: Mathematical Foundations of Information Theory, Dover Publications, New York, 1957.

Kletzing, C. A., Scudder, J. D., Dors, E. E., and Curto, C.: Auroral source region: plasma properties of the high latitude plasma sheet, J. Geophys. Res., 108, 1360, https://doi.org/10.1088/07413335/54/12/124001, 2003.

Kourakis, I., Sultana, S., and Hellberg, M. A.: Dynamical characteristics of solitary waves, shocks and envelope modes in kappa-distributed non-thermal plasmas: an overview, Plasma Phys. Contr. F., 54, 124001, https://doi.org/10.1088/07413335/54/12/124001, 2012.

Krimigis, S. M., Armstrong, T. P., Axford, W. I., Bostrom, C. O., Cheng, A. F., Gloeckler, G., Hamilton, D. C., Keath, E. P., Lanzerotti, L. J., Mauk, B. H., and Van Allen, J. A.: Hot plasma and energetic particles in Neptune's magnetosphere, Science, 246, 1483, 1989.

Laming, J. M., Moses, J. D., Ko, Y.-K., Ng, C. K., Rakowski, C. E., and Tylka, A. J.: On the remote detection of suprathermal ions in the solar corona and their role as seeds for solar energetic particle production, Astrophys. J., 770, 73, https://doi.org/10.1088/0004637X/770/1/73, 2013.

Le Roux, J. A., Webb, G. M., Shalchi, A., and Zank, G. P.: A generalized nonlinear guiding center theory for the collisionless anomalous perpendicular diffusion of cosmic rays, Astrophys. J., 716, 671-692, 2010.

Lee, E., Williams, D. R., and Lapenta, G.: Spectroscopic indication of suprathermal ions in the solar corona, arXiv:1305.2939v1, 2013.

Livadiotis, G.: Approach on Tsallis statistical interpretation of hydrogen-atom by adopting the generalized radial distribution function, J. Math. Chem., 45, 930-939, 2009.

Livadiotis, G.: Lagrangian temperature: derivation and physical meaning for systems described by kappa distributions, Entropy, 16, 4290-4308, 2014.

Livadiotis, G.: Statistical background and properties of kappa distributions in space plasmas, J. Geophys. Res., 120, 1607-1619, 2015a.

Livadiotis, G.: Kappa distribution in the presence of a potential energy, J. Geophys. Res., 120, 880-903, 2015 b.

Livadiotis, G.: Kappa and q indices: dependence on the degrees of freedom, Entropy, 17, 2062, https://doi.org/10.1209/02955075/113/10003, 2015c.

Livadiotis, G.: Curie law for systems described by kappa distributions, EPL-Europhys. Lett., 113, 10003, https://doi.org/10.1209/0295-5075/113/10003, 2016.

Livadiotis, G.: Kappa Distribution: Theory and Applications in Plasmas, Elsevier, the Netherlands, UK, US, 2017a. 
Livadiotis, G.: On the simplification of statistical mechanics for space plasmas, Entropy, 19, 285, https://doi.org/10.3390/e19060285, 2017b.

Livadiotis, G.: Using kappa distributions to identify the potential energy, J. Geophys. Res., https://doi.org/10.1002/2017JA024978, 2018.

Livadiotis, G. and McComas, D. J.: Beyond kappa distributions: exploiting Tsallis statistical mechanics in space plasmas, J. Geophys. Res., 114, A11105, https://doi.org/10.1029/2009JA014352, 2009.

Livadiotis, G. and McComas, D. J.: Exploring transitions of space plasmas out of equilibrium, Astrophys. J., 714, 971-987, 2010a.

Livadiotis, G. and McComas, D. J.: Measure of the departure of the $q$-metastable stationary states from equilibrium, Phys. Scripta, 82, 035003, https://doi.org/10.1088/0031-8949/82/03/035003, 2010b.

Livadiotis, G. and McComas, D. J.: The influence of pick-up ions on space plasma distributions, Astrophys. J., 738, 64, , https://doi.org/10.1088/0004-637X/738/1/64, 2011a.

Livadiotis, G. and McComas, D. J.: Invariant kappa distribution in space plasmas out of equilibrium, Astrophys. J., 741, 88, https://doi.org/10.1088/0004-637X/741/2/88, 2011 b.

Livadiotis, G. and McComas, D. J.: Non-equilibrium thermodynamic processes: space plasmas and the inner heliosheath, Astrophys. J., 749, 11, https://doi.org/10.1088/0004-637X/749/1/11, 2012.

Livadiotis, G. and McComas, D. J.: Understanding kappa distributions: a toolbox for space science and astrophysics, Space Sci. Rev., 75, 183-214, 2013a.

Livadiotis, G. and McComas, D. J.: Evidence of large scale phase space quantization in plasmas, Entropy, 15, 1118-1132, $2013 \mathrm{~b}$.

Livadiotis, G. and McComas, D. J.: Near-equilibrium heliosphere far-equilibrium heliosheath, AIP Conf. Proc., 1539, 344-350, 2013c.

Livadiotis, G. and McComas, D. J.: Electrostatic shielding in plasmas and the physical meaning of the Debye length, J. Plasma Phys., 80, 341-378, 2014.

Livadiotis, G., McComas, D.J, Dayeh, M. A., Funsten, H. O., and Schwadron, N. A.: First sky map of the inner heliosheath temperature using IBEX spectra, Astrophys. J., 734, 1, https://doi.org/10.1088/0004-637X/734/1/1, 2011.

Livadiotis, G., McComas, D. J., Randol, B., Möbius, E., Dayeh, M. A., Frisch, P. C., Funsten, H. O., Schwadron, N. A., and Zank, G. P.: Pick-up ion distributions and their influence on ENA spectral curvature, Astrophys. J., 751, 64, https://doi.org/10.1088/0004-637X/751/1/64/meta, 2012.

Livadiotis, G., McComas, D. J., Schwadron, N. A., Funsten, H. O., and Fuselier, S. A.: Pressure of the proton plasma in the inner heliosheath, Astrophys. J., 762, 134, https://doi.org/10.1088/0004637X/762/2/134, 2013.

Livadiotis, G., Assas., L., Dennis, B., Elaydi, S., and Kwessi, E.: A discrete time host-parasitoid model with an Allee effect, J. Biol. Dynam., 9, 34-51, 2015.

Livadiotis, G., Assas., L., Dennis, B., Elaydi, S., and Kwessi, E.: Kappa function as a unifying framework for discrete population modelling, Nat. Resour. Model., 29, 130-144, 2016.

Livi, R., Goldstein, J., Burch, J. L., Crary, F., Rymer, A. M., Mitchell, D. G., and Persoon, A. M.: Multi-instrument analysis of plasma parameters in Saturn's equatorial, inner mag- netosphere using corrections for spacecraft potential and penetrating background radiation, J. Geophys. Res., 119, 3683, https://doi.org/10.1002/2013JA019616, 2014.

Maksimovic, M., Pierrard, V., and Lemaire, J.: A kinetic model of the solar wind with Kappa distributions in the corona, Astron. Astrophys., 324, 725-734, 1997.

Malacarne, L. C., Mendes, R. S., and Lenzi, E. K.: Average entropy of a subsystem from its average Tsallis entropy, Phys. Rev. E, 65, 017106, https://doi.org/10.1103/PhysRevE.65.046131, 2001.

Mann, G., Classen, H. T., Keppler, E., and Roelof, E. C.: On electron acceleration at CIR related shock waves, Astron. Astrophys., 391, 749-756, 2002.

Mann, G., Warmuth, A., and Aurass, H.: Generation of highly energetic electrons at reconnection outflow shocks during solar flares, Astron. Astrophys., 494, 669-675, 2009.

Marsch, E.: Kinetic physics of the solar corona and solar wind, Living Rev. Sol. Phys., 3, 1, https://doi.org/10.12942/lrsp-2006-1, 2006.

Mauk, B. H., Krimigis, S. M., Keath, E. P., Cheng, A. F., Armstrong, T. P., Lanzerotti, L. J., Gloeckler, G., and Hamilton, D. C.: The hot plasma and radiation environment of the Uranian magnetosphere, J. Geophys. Res., 92, 15283, https://doi.org/10.1029/JA092iA13p15283, 1987.

Mauk, B. H., Mitchell, D. G., McEntire, R. W., Paranicas, C. P., Roelof, E. C., Williams, D. J., Krimigis, S. M., and Lagg, A.: Energetic ion characteristics and neutral gas interactions in Jupiter's magnetosphere, J. Geophys. Res., 109, A09S12, https://doi.org/10.1029/JA092iA13p15283, 2004.

Milovanov, A. V. and Zelenyi, L. M.: Functional background of the Tsallis entropy: "coarse-grained" systems and "kappa" distribution functions, Nonlin. Processes Geophys., 7, 211-221, https://doi.org/10.5194/npg-7-211-2000, 2000.

Moncuquet, M., Bagenal, F., and Meyer-Vernet, N.: Latitudinal structure of the outer Io plasma torus, J. Geophys. Res., 108, 1260, https://doi.org/10.1029/2001JA900124, 2002.

Montemurro, A.: Beyond the Zipf-Mandelbrot law in quantitative linguistics, Physica A, 300, 567-578, 2001.

Nicholls, D. C., Dopita, M. A., and Sutherland, R. S.: Resolving the electron temperature discrepancies in H II regions and planetary nebulae: $\kappa$-distributed electrons, Astrophys. J., 752, 148, https://doi.org/10.1088/0004-637X/752/2/148, 2012.

Nicholls, D. C., Dopita, M. A., Sutherland, R. S., Kewley, L. J., and Palay, E.: Measuring nebular temperatures: the effect of new collision strengths with equilibrium and $\kappa$ distributed electron energies, Astrophys. J. Suppl. S., 207, 21, https://doi.org/10.1088/0067-0049/207/2/21, 2013.

Nicolaou, G. and Livadiotis, G.: Misestimation of temperature when applying Maxwellian distributions to space plasmas described by kappa distributions, Astrophys. Space Sci., 361, 359, https://doi.org/10.1007/s10509-016-2949-z, 2016.

Ogasawara, K., Angelopoulos, V., Dayeh, M. A., Fuselier, S. A., Livadiotis, G., McComas, D. J., and McFadden, J. P.: Characterizing the dayside magnetosheath using ENAs: IBEX and THEMIS observations, J. Geophys. Res., 118, 3126-3137, 2013.

Ogasawara, K., Dayeh, M. A., Funsten, H. O., Fuselier, S. A., Livadiotis, G., and McComas, D. J.: Interplanetary magnetic field dependence of the suprathermal energetic neutral atoms originated in subsolar magnetopause, J. Geophys. Res., 120, 964-972, 2015. 
Ogasawara, K., Livadiotis, G., Grubbs, G. A., Jahn, J.-M., Michell, R., Samara, M., Sharber, J. R., and Winningham, J. D.: Properties of suprathermal electrons associated with discrete auroral arcs, Geophys. Res. Lett., 44, 3475-3484, 2017.

Olbert, S.: Summary of experimental results from M.I.T. detector on IMP-1, in: Physics of the Magnetosphere, edited by: Carovillano, R. L., McClay, J. F., and Radoski, H. R., Springer, New York, 641, 1968.

Ourabah, K., Ait Gougam, L., and Tribeche, M.: Nonthermal and suprathermal distributions as a consequence of superstatistics, Phys. Rev. E, 91, 012133, https://doi.org/10.1103/PhysRevE.91.012133, 2015.

Owocki, S. P. and Scudder, J. D.: The effect of a non-Maxwellian electron distribution on oxygen and iron ionization balances in the solar corona, Astrophys. J., 270, 758-768, 1983.

Pavlos, G. P., Malandraki, O. E., Pavlos, E. G., Iliopoulos, A. C., and Karakatsanis, L. P.: Non-extensive statistical analysis of magnetic field during the March 2012 ICME event using a multispacecraft approach, Physica A, 464, 149-181, 2016.

Pierrard, V. and Pieters, M.: Coronal heating and solar wind acceleration for electrons, protons, and minor ions, obtained from kinetic models based on kappa distributions, J. Geophys. Res., 119, 9441, https://doi.org/10.1002/2014JA020678, 2015.

Pierrard, V., Maksimovic, M., and Lemaire, J.: Electron velocity distribution function from the solar wind to the corona, J. Geophys. Res., 104, 17021-17032, 1999.

Pisarenko, N. F., Budnik, E. Yu., Ermolaev, Yu. I., Kirpichev, I. P., Lutsenko, V. N., Morozova, E. I., and Antonova, E. E.: The ion differential spectra in outer boundary of the ring current: November 17, 1995 case study, J. Atmos. Sol.-Terr. Phy., 64, 573-583, 2002.

Raadu, M. A. and Shafiq, M.: Test charge response for a dusty plasma with both grain size distribution and dynamical charging, Phys. Plasmas, 14, 012105, https://doi.org/10.1063/1.2431354, 2007.

Randol, B. M. and Christian, E. R.: Simulations of plasma obeying Coulomb's law and the formation of suprathermal ion tails in the solar wind, J. Geophys. Res., 119, 7025-7037, 2014.

Randol, B. M. and Christian, E. R.: Coupling of charged particles via Coulombic interactions: numerical simulations and resultant kappa-like velocity space distribution functions, J. Geophys. Res., 121, 1907-1919, 2016.

Raymond, J. C., Winkler, P. F., Blair, W. P., Lee, J.-J., and Park, S.: Non-Maxwellian $\mathrm{H} \alpha$ profiles in Tycho's supernova remnant, Astrophys. J., 712, 901, https://doi.org/10.1086/589645, 2010.

Rubab, N. and Murtaza, G.: Debye length in non-Maxwellian plasmas, Phys. Scripta, 74, 145, https://doi.org/10.1088/00318949/74/2/001, 2006.

Ruseckas, J.: Probabilistic model of $\mathrm{N}$ correlated binary random variables and non-extensive statistical mechanics, Phys. Lett. A, 379, 654-659, 2015.

Saito, S., Forme, F. R. E., Buchert, S. C., Nozawa, S., and Fujii, R.: Effects of a kappa distribution function of electrons on incoherent scatter spectra, Ann. Geophys., 18, 1216-1223, https://doi.org/10.1007/s00585-000-1216-2, 2000.

Salazar, R. and Toral, R.: Scaling laws for a system with long-range interactions within Tsallis statistics, Phys. Rev. Lett., 83, 42334236, 1999.
Shannon, C. E.: A mathematical theory of communication, Bell Syst. Tech. J., 27, 379-423, 623-656, 1948.

Silva, R., Plastino, A. R., and Lima, J. A. S.: A Maxwellian path to the q-nonextensive velocity distribution function, Phys. Lett. A, 249, 401-408, 1998.

Štverák, S., Maksimovic, M., Travnicek, P. M., Marsch, E., Fazakerley, A. N., and Scime, E. E.: Radial evolution of nonthermal electron populations in the low-latitude solar wind: Helios, Cluster, and Ulysses observations, J. Geophys. Res., 114, A05104, https://doi.org/10.1029/2008JA013883, 2009.

Tirnakli, U. and Borges, E. P.: The standard map: from BoltzmannGibbs statistics to Tsallis statistics, Sci. Rep.-UK, 6, 23644, https://doi.org/10.1038/srep23644, 2016.

Tribeche, M., Mayout, S., and Amour, R.: Effect of ion suprathermality on arbitrary amplitude dust acoustic waves in a charge varying dusty plasma, Phys. Plasmas, 16, 043706, https://doi.org/10.1063/1.3118592, 2009.

Tsallis, C.: Possible generalization of Boltzmann-Gibbs statistics, J. Stat. Phys., 52, 479-487, 1988.

Tsallis, C.: Introduction to Nonextensive Statistical Mechanics, Springer, New York, 2009.

Tsallis, C. and De Albuquerque, M. P.: Are citations of scientific papers a case of nonextensivity, Eur. Phys. J. B, 13, 777-780, 2000.

Tsallis, C., Gell-Mann, M., and Sato, Y.: Asymptotically scaleinvariant occupancy of phase space makes the entropy Sq extensive, P. Natl. Acad. Sci. USA, 102, 15377-15382, 2005.

Umarov, S., Tsallis, C., and Steinberg, S.: On a $q$-central limit theorem consistent with nonextensive statistical mechanics, Milan J. Math., 76, 307, https://doi.org/10.1007/s00032-008-0087$\mathrm{y}, 2008$

Varotsos, P. A., Sarlis, N. V., and Skordas, E. S.: Study of the temporal correlations in the magnitude time series before major earthquakes in Japan, J. Geophys. Res., 119, 9192-9206, 2014.

Vasyliũnas, V. M.: A survey of low-energy electrons in the evening sector of the magnetosphere with OGO 1 and OGO 3, J. Geophys. Res., 73, 2839-2884, 1968.

Villain, J.: On the long-range interactions and non-extensive systems, Scientifica Acta, 2, 93-99, 2008.

Viñas, A. F., Moya, P. S., Navarro, R., and Araneda, J. A.: The role of higher-order modes on the electromagnetic whistler-cyclotron wave fluctuations of thermal and non-thermal plasmas, Phys. Plasmas, 21, 012902, https://doi.org/10.1063/1.4861865, 2014.

Viñas, A. F., Moya, P. S., Navarro, R. E., Valdivia, J. A., Araneda, J. A., and Muñoz, V.: Electromagnetic fluctuations of the whistler-cyclotron and firehose instabilities in a Maxwellian and Tsallis-kappa-like plasma, J. Geophys. Res., 120, 3307 3317, 2015.

Vocks, C., Mann, G., and Rausche, G.: Formation of suprathermal electron distributions in the quiet solar corona, Astron. Astrophys., 480, 527-536, 2008.

Wang, C.-P., Lyons, L. R., Chen, M. W., Wolf, R. A., and Toffoletto, F. R.: Modeling the inner plasma sheet protons and magnetic field under enhanced convection, J. Geophys. Res., 108, 1074, https://doi.org/10.1029/2002JA009620, 2003.

Xiao, F., Shen, C., Wang, Y., Zheng, H., and Whang, S.: Energetic electron distributions fitted with a kappa-type function at geosynchronous orbit, J. Geophys. Res., 113, A05203, https://doi.org/10.1088/0741-3335/50/6/062001, 2008. 
Yamano, T.: Some properties of $q$-logarithmic and $q$-exponential functions in Tsallis statistics, Physica A, 305, 486-496, 2002.

Yoon, P. H.: Electron kappa distribution and quasi-thermal noise, J. Geophys. Res., 119, 7074-7087, 2014.

Yoon, P. H., Rhee, T., and Ryu, C. M.: Self-consistent formation of electron $\kappa$ distribution: 1. Theory, J. Geophys. Res., 111, A09106, https://doi.org/10.1029/2006JA011681, 2006.

Yoon, P. H., Ziebell, L. F., Gaelzer, R., Lin, R. P., and Wang, L.: Langmuir turbulence and suprathermal electrons, Space Sci. Rev., 173, 459-489, 2012.

Zank, G. P.: Faltering steps into the galaxy: the boundary regions of the heliosphere, Annu. Rev. Astron. Astr., 53, 449, https://doi.org/10.1029/2006JA011681, 2015.

Zank, G. P., Heerikhuisen, J., Pogorelov, N. V., Burrows, R., and McComas, D. J.: Microstructure of the heliospheric termination shock: implications for energetic neutral atom observations, Astrophys. J., 708, 1092, https://doi.org/10.1088/0004637X/708/2/1092, 2010.
Zhang, Y., Liu, X.-W., and Zhang, B.: H-I free-bound emission of planetary nebulae with large abundance discrepancies: twocomponent models vs. $\kappa$-distributed electrons, Astrophys. J., 780, 93, https://doi.org/10.1088/0004-637X/780/1/93., 2014.

Zirnstein, E. J. and McComas, D. J.: Using kappa functions to characterize outer heliosphere proton distributions in the presence of charge-exchange, Astrophys. J., 815, 31, https://doi.org/10.1088/0004-637X/815/1/31, 2015.

Zouganelis, I.: Measuring suprathermal electron parameters in space plasmas: implementation of the quasi-thermal noise spectroscopy with kappa distributions using in situ Ulysses/URAP radio measurements in the solar wind, J. Geophys. Res., 113, A08111, https://doi.org/10.1029/2007JA012979, 2008. 